\title{
An approach to a cross layer-based QoE improvement for MANET routing protocols
}

\author{
Ramon Sanchez-Iborra, Maria-Dolores Cano \\ Dept. of Information Technologies and Communications, Polytechnic University of \\ Cartagena \\ Antiguo Cuartel de Antigones. Plaza del Hospital, No 1, 30202 Cartagena (Murcia) \\ Tel: +34 $968338872 \quad$ E-mail: \{ramon.sanchez, mdolores.cano\} @ upct.es
}

Received: March 27, 2014

Accepted: June 20, 2014

Published: August 14, 2014

DOI: $10.5296 /$ npa.v6i3.5827

URL: http://dx.doi.org/10.5296/npa.v6i3.5827

\begin{abstract}
Currently, multimedia communications are monopolizing Internet traffic. These services present highly demanding real-time requirements that need great bandwidths in order to provide acceptable quality levels. Meanwhile, the variety of network architectures employed by final users to access these contents is wider and wider. One of the most attractive access networks due to its ease-of-deployment and decentralized architecture is the Mobile Ad-hoc NETworks (MANETs). The inherent dynamic nature of these networks entails the use of appropriate routing protocols in order to obtain good performance in the services supported by the system. In this work, we focus on evaluating the performance of the novel routing protocol Better Approach To Mobile Ad-hoc Networking (BATMAN) and the widely extended IETF experimental drafts Optimized Link State Routing (OLSR) and Ad hoc On-Demand Distance Vector (AODV) protocols supporting video traffic. The results obtained from a chain-topology network have been improved in terms of Quality of Experience (QoE) by means of different modifications at different OSI layers. We have found out that each protocol presents low performance in different scenarios that can be enhanced by means of the proposed techniques. As we show in this work, taking a proper action in the right OSI layer permits an improvement on the multimedia traffic support in MANETs.
\end{abstract}

Keywords: BATMAN; OLSR; AODV; MANETs; QoS; QoE; Video transmission 


\section{Introduction}

Nowadays, multimedia communications are attracting great attention from the content providers, telcos, and final users. The latter are currently consuming these services from many different access networks and architectures. Certainly, wireless networks are those which provide the most attractive features to customers, such as mobility, or configuration and deployment simplicity. Focusing on the most extended wireless standard, namely IEEE 802.11, it can adopt two different architecture schemes, namely, infrastructure and ad-hoc modes. The former consists of a central point, e.g., a router, which manages the network, assuming IP assignment, routing tasks, etc. Several works have proved the validity of this mode to support multimedia traffic, such as Voice over IP (VoIP) [1], [2] or video transmission [3]. These works evaluated the impact of several parameters, such as packet loss rate, delay, or fading channels, on the quality obtained in different types of multimedia communications under different conditions, scenarios, etc., concluding that the IEEE 802.11 infrastructure mode is suitable to support multimedia traffic with good levels of quality.

On the other hand the ad-hoc mode has received less attention by the research community in terms of evaluating its capacity to support multimedia traffic. Networks formed by nodes in ad-hoc mode (MANETs, Mobile Ad-hoc NETworks) present interesting characteristics for different situations, especially for those in which it is necessary the quick deployment of a temporary network. MANETs make use of a completely decentralized scheme, in which all nodes forming the networks assume both, host and router roles, which permits the deployment of a network without a central point or a pre-existent infrastructure. Moreover, while an infrastructure network is limited to the coverage range of the central point, a MANET could be extended by the coverage range of the nodes in the system, permitting a great mobility by the nodes within it, too. Due to the dynamic nature of these networks, they are able to auto-configure themselves, reacting to changes in their topology. However, the transmission of multimedia traffic entails real-time constraints, necessary to provide these services with acceptable levels of quality. For that reason, efficient routing protocols are needed, capable of setting the paths between nodes as fast as possible and with the ability of reacting to scenario changes.

In this work, we evaluate through computer-simulation, the performance of three of the most notable routing protocols, namely, BATMAN (Better Approach To Mobile Ad-hoc Networking) [4], OLSR (Optimized Link State Routing) [5], and AODV (Ad hoc On-Demand Distance Vector) [6] managing MANETs supporting multimedia traffic. The most recently proposed algorithm, BATMAN, which is still defined as an IETF draft is a promising protocol that has attracted great attention during the last times [7], [8]. Meanwhile, OLSR and AODV, whose status are denoted as experimental RFCs by the IETF, have been more analyzed than BATMAN, showing better performance than other ad-hoc routing protocols in multimedia-delivery scenarios [9], [10]. Specifically, we carry out a video transmission Quality of Service (QoS) and Quality of Experience (QoE) evaluation in a MANET with chain-topology managed by the aforementioned routing protocols. For video coding, we employed the widely used H.264-SVC coding scheme. Additionally, we analyze the impact of the PHY layer on the communications quality, by characterizing the wireless 
medium with the Nakagami-m model, which permit us to evaluate the effect of the fading channels on the multimedia stream. The results obtained in the most adverse scenarios are compared with those obtained in Free Space environments. As the most important contribution, we propose several actions that can be taken from different OSI layers in order to enhance the video communications quality. Thus, we demonstrate that the improvement of quality, no matter the system under study, should be considered from a cross-layer perspective, since the binding of different-layer techniques allows gathering the contribution of each of them; thus, the desired increase in the quality-level of the provided service can be achieved more successfully than just focusing on optimizing one single parameter.

The rest of paper is organized as follows. Section II presents a review of the related literature, focusing in those works that evaluate the performance of ad-hoc routing protocols supporting multimedia services. Section III describes the main characteristics of the routing protocols under study, BATMAN, OLSR, and AODV. A description of the simulation environment employed in this work is included in Section IV. Section V shows the results achieved and discusses the response of the three different routing protocols to the several proposals from different OSI layers that are presented, aiming the improvement of the video QoE. Conclusions are drawn in Section VI.

\section{Related work}

As aforementioned, the suitability of MANETs to support multimedia services has not been as investigated as it occurs for infrastructure networks. However, some works analyzing the performance of ad-hoc systems supporting multimedia communications can be found in the literature [7], [8], [11], [12].

Reineri et al. have studied the performance of BATMAN, OLSR, and AODV supporting multimedia traffic in mobility scenarios [7], [8]. In [7], authors noticed some problems in the case of BATMAN for downlink traffic transfer, due to its low reactivity when topology changes occur. Thus, they proposed a new scheme for the window-mechanism employed by BATMAN in order to estimate the quality of the paths, obtaining very promising results. On the other hand, work in [8] presented a performance evaluation of different ad-hoc routing protocols, including their enhanced version of BATMAN, in a dynamic real test-bed. Authors found that the improved version of BATMAN outperforms OLSR and the standard BATMAN in terms of percentage of received video-traffic and jitter, making possible video-streaming communications with low number of hops between communicants. A quite similar work was presented in [11], in which authors evaluated the performance of BATMAN supporting multimedia traffic (audio and video) in static real test-beds. In their results, Kulla et al. demonstrated the capability of MANETs formed by few nodes to support VoIP traffic; however, the limitations to support more demanding traffic, such as video-streaming were also manifested. Finally, Adam et al. also presented a comparison among OLSR, AODV, and DSR (Dynamic Source Routing) [12]. In this work, authors carried out a performance 
evaluation of these protocols supporting CBR (Constant Bit Rate) traffic emulating a multimedia service. They found out that AODV beats OLSR in terms of jitter and Packet Delivery Ratio (PDR), but with rather high routing overhead. On the other hand, OLSR presented better performance than AODV in terms of delay with a high number of simultaneous connections in the network.

Given this inconclusive results about which routing protocol is better suited to support multimedia traffic, we present a multi-hop network composed by up to 10 intermediate nodes and up to 5 simultaneous realistic video transmissions. The latter have been characterized by a video-trace extracted from a real video-signal instead of employing simple CBR traffic. This configuration has permitted us to evaluate the effect of the interferences or the excess of control packets in the network generated by a great amount of nodes in the system interchanging Variable Bit Rate (VBR) traffic.

\section{Routing protocols}

The routing protocol in charge of managing a MANET is the main agent that permits the good performance of the services working over the network. Depending on the features and constraints of these services, the election of one or another routing protocol is a crucial decision that will be conditioned by the own characteristics of the routing algorithm. One extended classification to sort routing protocols is differentiating them into (i) pro-active, (ii) reactive, or (iii) hybrid protocols.

The main feature of pro-active protocols is that all the nodes maintain routing information even before it is needed. This is achieved by a periodical interchange of control messages between nodes. The principal drawback of this type of protocols is the great amount of routing messages through the network, which provoke a decrease on the performance of the system, for instance, dropping the nodes throughput. Moreover, pro-active protocols present some scalability issues in high populated networks, due to the increasing amount of control information that nodes have to manage. On the other hand, the nodes are always ready to the fast retransmission of the incoming packets to the next hop in the route due to the updated network information that they keep. This feature allows reducing the transmission delay, improving the performance of time-sensitive services. Examples of proactive protocols are DSDV (Destination-Sequenced Distance Vector), BATMAN, or OLSR.

Reactive protocols, also known as on-demand protocols, just calculate the route from a node to another when it is necessary. In other words, there is no control information travelling through the network if there is not any current connection between nodes. When a node wants to communicate with another one, then the route is looked-up on-demand and the connection is established. That scheme provides a reduction on the bandwidth employed for routing tasks, but could increase the latency of packet-retransmissions, harming the quality of real-time applications. Examples of on-demand algorithms are TORA (Temporally Ordered Routing Algorithm), DYMO (Dynamic Manet On-demand routing), or AODV.

Finally, hybrid protocols combine the strengths of proactive and on-demand algorithms. 
These protocols usually organize the network hierarchically, i. e., dividing the system in zones managed proactively and others controlled by means of reactive approaches. One of the most extended hybrid protocols is the Zone Routing Protocol (ZRP). Next we present a brief description of the protocols under study, BATMAN, OLSR, and AODV.

\section{A. BATMAN}

This proactive protocol is under development by the "Freifunk Community" [13]. The novelty of BATMAN resides in the decentralization of the knowledge about the routes; in other words, single nodes do not have routing tables for the entire network. Instead, each node determines one single-hop neighbor for each destination in the mesh, which can be utilized as the best gateway to communicate with the destination node. Thus, a very fast and efficient routing scheme is developed, creating a network of collective intelligence, and allowing low CPU and consequently less battery consumption for each node [14]. The protocol operation is as follows. On a regular basis every node broadcasts an OriGinator Message (OGM), thereby informing its link-local neighbors about its existence. Link-local neighbors which are receiving the OGM messages are relaying them by rebroadcasting, according to the specific BATMAN forwarding rules. The BATMAN mesh network is therefore flooded with OGM messages until every node has received each of them at least once, or until they got lost due to packet loss occurred in the communication links, or until their TTL value has expired. The number of OGM messages received from a given node via each link-local neighbor is used to estimate the quality of a route. In order to be able to find the best route to a particular end node, BATMAN counts the OGM messages received from each node in the network and logs which link-local neighbor relayed the message. Using this information BATMAN maintains a table with the best link-local route towards every other node in the network. By using a sequence number, included in each OGM, BATMAN can distinguish between new OGM packets and their duplicates, ensuring that every OGM gets only counted once. Notice that OGMs should not be taken actually as routing-information interchange packets, as they only act as a "hello packet", not containing any information about routing tables, link states, etc.

\section{B. OLSR}

This routing algorithm for MANETs is a well-known IETF's experimental-draft, based on the traditional concept of link-state routing algorithm. OLSR makes use of two different types of control packets, namely, HELLO and Topology Control (TC) packets. HELLO packets are employed by nodes to find out their neighboring nodes, so these packets are not retransmitted to the entire network. Once each node knows its "neighborhood", it starts sending TC packets including its neighbors and the state of the links established between them. This helps other nodes to build the network topology. The improvement introduced by OLSR is that the amount of control traffic, specifically TC packets, in the network is reduced by employing the Multi-Point Replaying (MPR) strategy. To do this, each node selects a set of its neighbors to retransmit its TC packets, including them in a field within the packet header; the remaining 
nodes can read these packets but are not allowed to retransmit them. With a proper strategy selecting the re-transmitter neighbors, all the destinations are reachable by all the nodes in the system without flooding the entire network. Additionally, TC packets include a sequence number in order to avoid infinite retransmissions due to undesirable loops.

\section{C. $A O D V$}

This reactive ad-hoc routing protocol is based on the Bellman-Ford algorithm. Following the typical procedure of the on-demand approaches, AODV just maintains the active routes. These paths are kept at a next-hop routing table stored by each node, which contains the next single-hop neighbor in the route to a given destination. In order to save storage capacity and to ensure the freshness of the routes, the routing table entries are removed after a specified inactivity time. When a source node wants to communicate with another one, first it looks for an active path to the destination in its routing table. If no route is found, the source node initiates a "route discovery" operation. This process begins with the broadcast of a Route REQuest (RREQ) packet. The RREQ, which includes the source and destination addresses, is re-broadcasted until it reaches the destination or a node that knows a path to the destination. To avoid an excessive flooding of control packets, they include a unique ID that permits the nodes to discard duplicate RREQ packets. Once a node receives an RREQ, it keeps the route back to the source and if the destination is not known, the RREQ is retransmitted to its neighbors; otherwise, it sends back to the destination a Route REPly (RREP) packet. All the intermediate nodes that receive this message update its next-hop table with respect to the destination node and retransmit the RREP to the next hop to the source. Finally, when the source receives the RREP packet, the path is established and the communication begins. In order to monitor the status of direct links, nodes send periodically "hello" messages. When a link disconnection is discover, it is announced by a Router ERRor (RERR) message to the full network.

\section{Simulation environment}

The employed platform for the simulation study was Omnet++ v4.4, with the InetManet framework in its version 2.2 [15]. These libraries provide the description of different-layer communication protocols implemented in several network devices. As explained above, the simulated scenario is an IEEE 802.11g (WiFi) network at $54 \mathrm{Mbps}$ in ad-hoc mode. Channel access is performed according to the Distributed Coordination Function (DCF) scheme. We use the topology shown in Fig. 1: a static chain of nodes with a variable number of hops between video transmitters (TX) and video receivers (RX). The nodes are located with a distance of $100 \mathrm{~m}$ between them; with that configuration, each node is only capable to establish a link with its direct neighbors, avoiding the appearance of shortcuts when calculating the route between the extremes of the communication. This configuration allow us (i) to evaluate the impact of the number of hops between communicants on the quality of the transmission, and (ii) to analyze the maximum load that one node is capable to support since all the transmitters and receivers connect directly with one or the other extreme-node of 
the chain. We have carried out 10 simulation instances with different seeds for every evaluated scenario. Thereby, our results are obtained by taking the average value for every parameter under evaluation avoiding non representative values. We have also calculated the variance and confidence interval for every measurement, but they are not shown due to their low significance. The wireless card transmission power is set to $20 \mathrm{~mW}$, which is a typical value for different-brand wireless cards, such as the Cisco Aironet Series. Following the specifications of typical commercial cards, we set the cards sensitivity to $-72 \mathrm{dBm}$, with a carrier frequency of $2.4 \mathrm{GHz}$ (802.11g standard), and a SNIR (Signal to Interference and Noise Ratio) threshold of $4 \mathrm{~dB}$. The resulting transmission range employing the described parameters and characterizing the wireless transmission medium with the Free Space model is $175 \mathrm{~m}$.

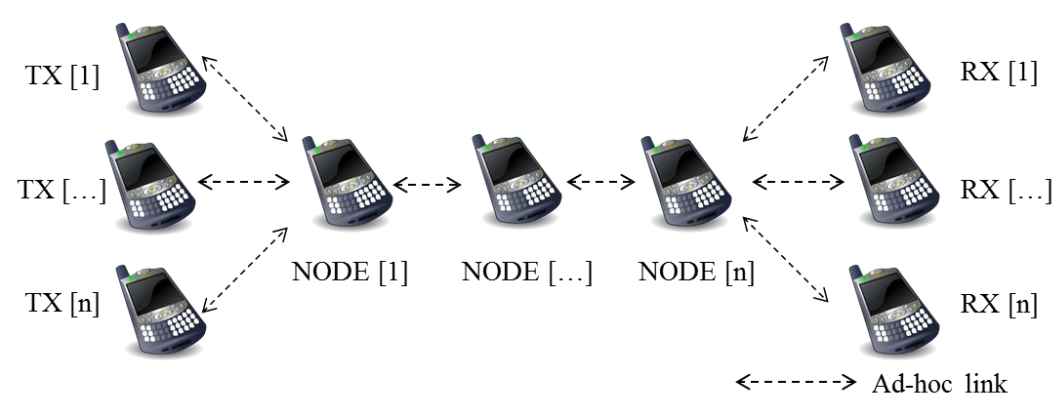

Figure 1. Ad-hoc chain topology.

Regarding video transmission, we have employed the UDPVideoStreamCli2 and the UDPVideoStreamSvr2 modules. These UDP apps emulate a video-stream communication in which the video server sends to the client UDP-traffic following a typical pattern for a video transmission. In our test-bench we have employed a realistic video-trace available in [16], corresponding to a video encoded at 30 frames per second (fps) with the H.264/SVC single layer VBR (Variable Bit Rate) codec. The frame size is 352 x 288 px (CIF format), the GoP (Group of Pictures) is 16, the number of B frames between I and P frames is 1 (G16B1). Given these video features, the encoding system generates VBR frames with an average size of 485.5 Bytes, transmitting them at $118 \mathrm{Kbps}$. Regarding quality measurement, we have employed both QoS and QoE metrics. The latter have been calculated in terms of MOS (Mean Opinion Score), from the parametric model presented in [17]. As shown in expression (1), this model allows us to obtain accurate video-MOS estimations by means of the Packet Loss Rate (PLR) and the video-coding bit-rate (Br) parameters.

$V_{q}=1+\left(v_{1}-\frac{v_{1}}{1+\left(\frac{B r}{v_{2}}\right)^{v_{3}}}\right) e^{-\frac{P L R}{v_{4}}}$

The MOS-estimation expression (1) makes use of the experimental factors v1, v2, v3, and v4, which are dependent of the coding scheme employed. The starting time for each multimedia communication is chosen randomly according to a Poisson distribution in a time range of $(0$, $10 \mathrm{~s}$ ), after the convergence time for each routing protocol. The video sources last $30 \mathrm{~s}$ and 
the queue size at the MAC layer for each node in the network is 50 frames. The remaining 802.11g parameters, such as DIFS (DCF Interframe Space), SIFS (Short Interframe Space), slot time, contention window $\left(\mathrm{CW}_{\mathrm{MIN}}\right)$, and different-layers headers are shown in Table 1, which additionally includes the main routing protocols time-outs, too.

Finally, we have modeled the wireless transmission channel as realistic as possible in order to avoid inaccuracies introduced by the simulation platform. We consider the effect of the fading channels on the wireless transmission medium by comparing the results obtained under Free Space conditions with those attained in scenarios characterized by the well-known Nakagami-m model, which has been shaped with an $m$ value of 5 .

Table $1.802 .11 \mathrm{~g}$ parameters and routing protocols time-intervals

\begin{tabular}{|c|c|c|}
\hline Parameters & Bytes & Time \\
\hline \hline SIFS, DIFS, SLOT $(\mu \mathrm{s})$ & - & $\{10,28,9\}$ \\
\hline CW $_{\text {MIN }}($ slots $)$ & - & 31 \\
\hline PLCP preamble $(\mu \mathrm{s})$ & $-, 28,8$ & $416,4.15,1.18\}$ \\
\hline$\{$ PLCP, MAC, SNAP $\}$ headers $(\mu \mathrm{s})$ & 40 & 5.92 \\
\hline IP + UDP + RTP headers $(\mu \mathrm{s})$ & 14 & 2.07 \\
\hline ACK $(\mu \mathrm{s})$ & - & 1 \\
\hline BATMAN OGM interval $(\mathrm{s})$ & - & 200 \\
\hline BATMAN purge timeout $(\mathrm{s})$ & - & $\{2,5,5\}$ \\
\hline OLSR $\{$ HELLO, TC, MID $\}$ intervals $(\mathrm{s})$ & - & 3 \\
\hline OLSR $\{$ Neighbor, Topology $\}$ hold times $(\mathrm{s})$ & - & 1 \\
\hline AODV Active Route timeout $(\mathrm{s})$ & - & $35\}$ \\
\hline AODV Hello interval $(\mathrm{s})$ & - & \\
\hline
\end{tabular}

\section{Results}

In this section we present the obtained results in order to evaluate the performance of the multimedia delivery system presented in previous sections. We focus on analyzing the impact of several parameters, e.g., fading channels, number of hops, and number of simultaneous multimedia streams, on the quality of the transmissions attained in the chain network (see Fig. 1) managed by the three routing protocols under study, with their configuration parameters by default. To this end, we have collected different QoS/QoE metrics, such as delay, PLR, PDR, and Mean Opinion Score (MOS). Then, we show a series of actions that have been taken from different OSI layers, which permit an improvement in the routing protocols performance and, therefore, in the video quality.

\section{A. Regular evaluation}

In order to evaluate the quality of video transmissions, several metrics have been proposed in the bibliography, namely, MOS, latency, PDR, etc. Following the guidelines of ITU-T recommendations G.114 and G.1010, we define a video-transmission as valid if the final MOS value obtained for this communication is over 3.4. Thus, we gather the impact of many network impairments into one parameter. Additionally, from a QoS perspective, the 


\section{Macrothink Institute ${ }^{\mathrm{TM}}$}

maximum PLR is $1.5 \%$. As described before, we have obtained the video MOS estimations by the use of the parametric model presented in [17]. Fig. 2 shows a comparison between the MOS obtained for different number of simultaneous video-streams delivered through the system configured with several multi-hop configurations. Regarding the impact of the fading channels, observe the deep drop suffered by the MOS in the Nakagami-m scenarios managed by OLSR (Fig. 2.d) and AODV (Fig. 2.f) in comparison with the MOS levels in Free Space environments (Fig. 2.c and Fig. 2.e): disregarding the number of hops between TX and RX, no streams are accepted as valid by the system in the most adverse environment (all the MOS values are under 3.4).

On the other hand, the MOS fall in fading scenarios is less pronounced using BATMAN (please compare Fig. 2.b, Fig. 2.d, and Fig. 2.f). BATMAN accepts up to 5 simultaneous transmissions with 2 hops between TXs and RXs. Moreover, lower number of video-streams also achieves high levels of quality with greater amount of intermediate nodes separating TX and RX. We ascribe the better performance of BATMAN in hostile scenarios to the different routing-information dissemination approach employed by the three protocols under consideration. While BATMAN employs a great amount of small OGM packets as control information, OLSR and AODV perform their control-messages interchange by means of lower number of larger packets, which are more prone to the impairments introduced by the wireless transmission channel. Therefore, we can conclude, that BATMAN is more robust than OLSR and AODV in adverse channel conditions.

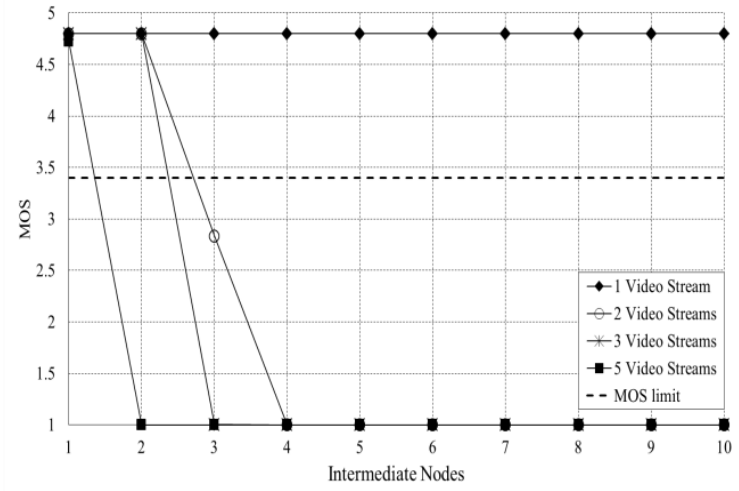

(a) BATMAN. Free Space

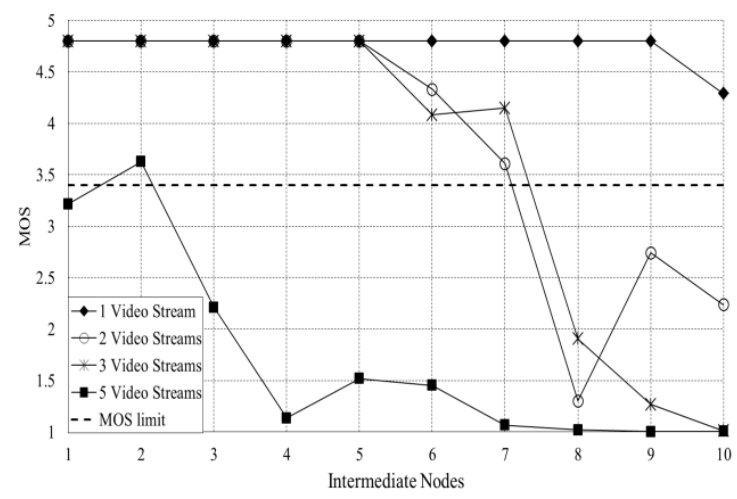

(c) OLSR. Free Space

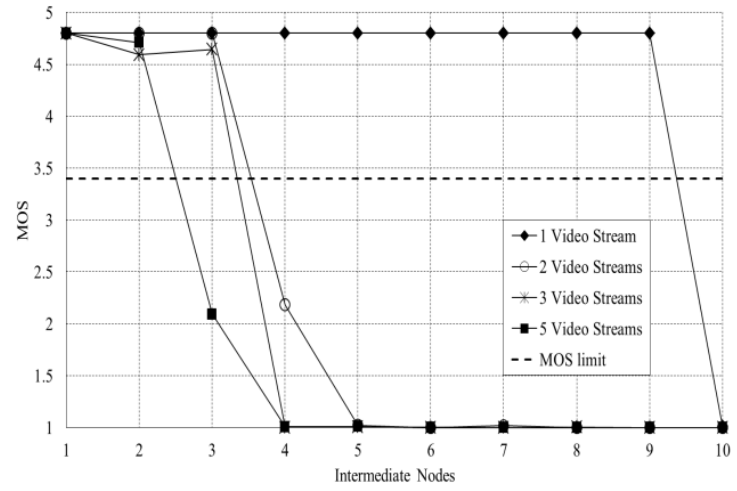

(b) BATMAN. Nakagami-m

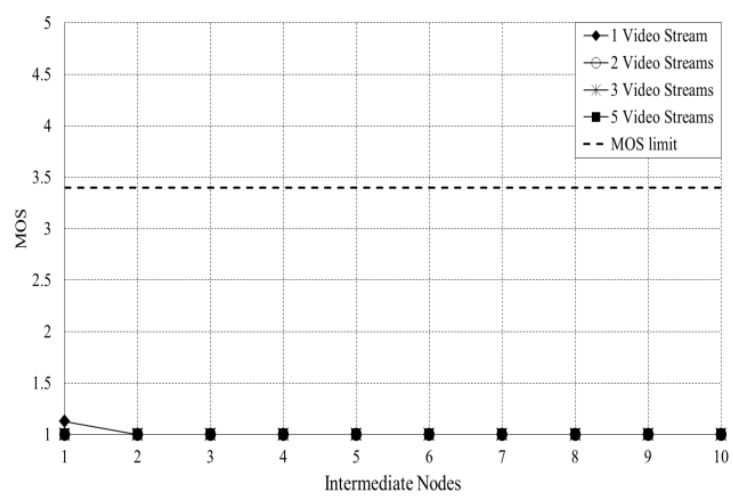

(d) OLSR. Nakagami-m 


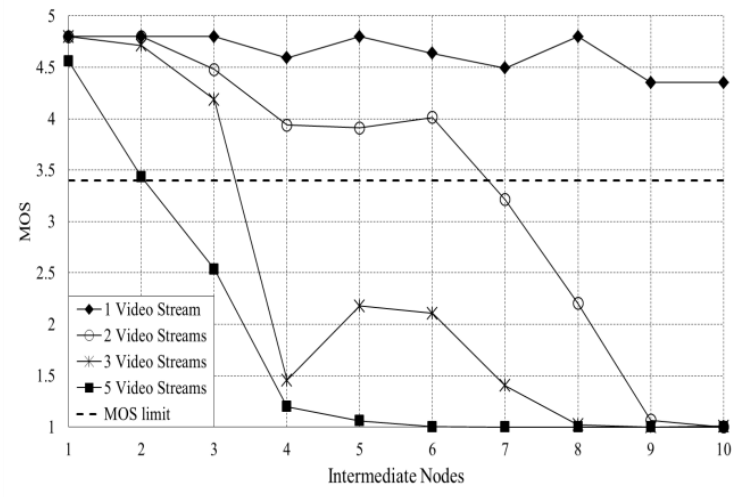

(e) AODV. Free Space

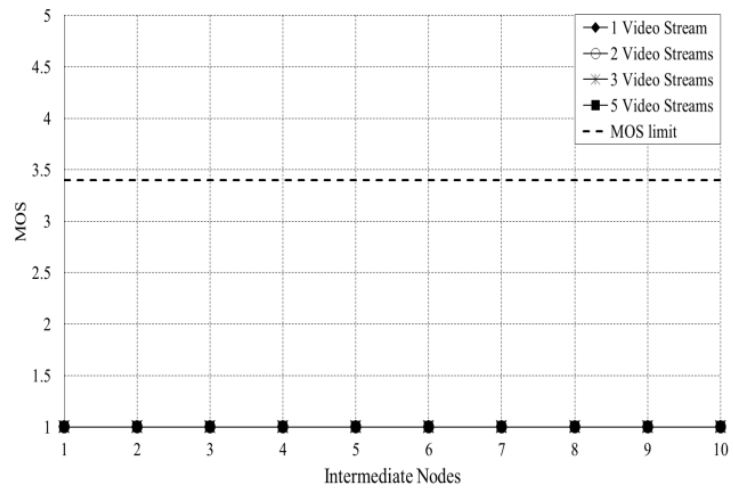

(f) AODV. Nakagami-m

Figure 2. MOS attained for a variable number of video-streams and hops between transmitters and receivers. Results for BATMAN in Free

Space (a) and Nakagami-m scenarios (b), OLSR in Free Space (c) and Nakagami-m scenarios (d), and AODV in Free Space (e) and

Nakagami-m scenarios (f).

Nevertheless, OLSR over performs BATMAN in the scenarios characterized by the Free Space propagation model (see Fig. 2.a and 2.c). Using OLSR, the system accepts up to 3 simultaneous streams in a 7 hops connection. However, only one video-stream is accepted as valid with BATMAN when there are more than 2 hops between TX and RX. Similarly, the system just allows up to 3 simultaneous transmissions when the distance between video communicants is 2 hops. Observing Fig. 2.a and Fig. 2.b, it is clear that BATMAN behaves better in a scenario with fading than in Free Space; this outcome will be discussed in next sub-sections. Meanwhile, AODV presents a similar poor performance to OLSR in Nakagami-m scenarios, and beats BATMAN in Free Space, but far from the MOS values attained with OLSR. As discussed previously, the reactive protocols are less suited than the proactive ones to support delay-sensitive services, such as video-streaming, and the obtained MOS is lower than that obtained with the other protocols, even with low number of simultaneous video-streams in the network (see Fig. 2.e and Fig. 2.f).

This overall behavior can also be observed from a QoS perspective. In order to simplify the obtained outcomes, we present as an example the PLR obtained for two simultaneous video-streams crossing the chain topology managed by the three routing protocols under consideration (see Fig. 3). Observe the almost optimal results obtained for OLSR in Free Space scenarios, with the PLR near $0 \%$ in every scenario, except in those with the greatest number of nodes separating both extremes of the communication ( 9 and 10 intermediate nodes). On the other hand, the worst results are obtained by this same protocol in the scenarios characterized by the Nakagami-m propagation model. It is also remarkable the great level of packet loss obtained by AODV in Nakagami-m scenarios, which is consistent with the poor MOS estimated previously. 


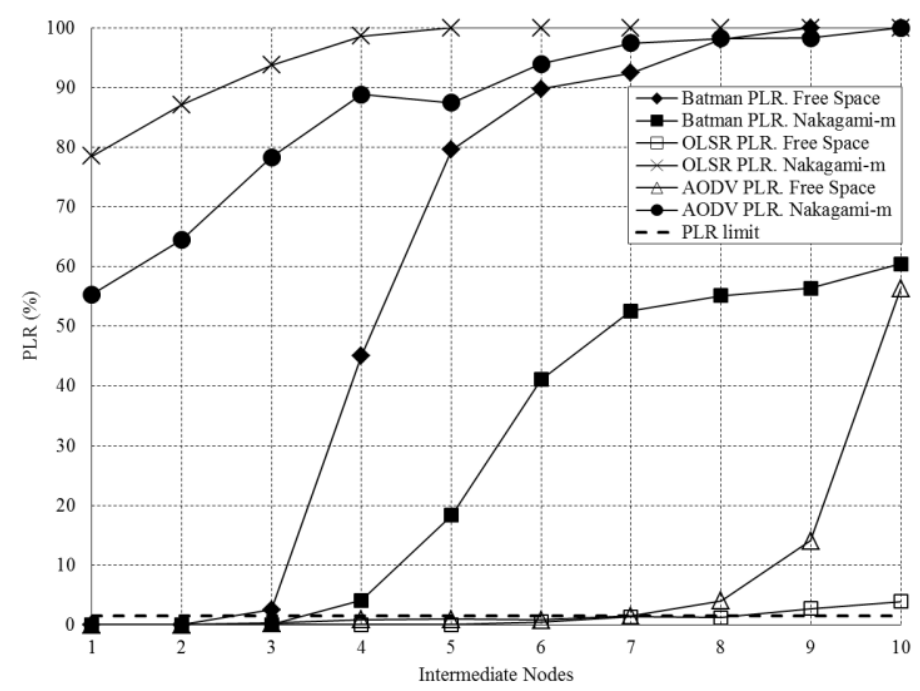

Figure 3. Packet loss rate suffered by the video-streams in chain scenario with two simultaneous flows and variable number of hops between transmitters and receivers, using BATMAN, OLSR, and AODV.

\section{B. Improvement from the Physical Layer}

As discussed above, the medium hostility usually impacts negatively in the performance of the system. In the previous sub-section we showed the poor performance of both OLSR and AODV in scenarios characterized by the Nakagami-m propagation model. One action that can be taken from the lower level of the OSI stack is tuning properly the transmission power of the wireless cards, since other parameters, such as the sensitivity, SNiR, etc. are not modifiable. As shown in Fig. 4, by increasing the transmission power of the nodes, we achieve a clear improvement in the MOS for the three protocols evaluated. This action may consist on tuning the transmission power taking into account the levels of quality and SNiR measured at real-time during the transmission. Thus, the balance between transmission power and battery consumption can be considered. Fig. 4 depicts the MOS evolution for the most hostile scenario (Nakagami-m) with 2 simultaneous video-streams managed by BATMAN, OLSR, and AODV employing different transmission powers. Observe how OLSR and AODV, improve their performance with the increase on the transmission power. However, we obtain a surprising result when employing BATMAN. The optimal result for this protocol is obtained for a transmission power of $100 \mathrm{~mW}$ (Fig. 4.b); on the other hand, further increasing in the transmitters power provokes, first the system instability at $300 \mathrm{~mW}$ (Fig. 4.c) and, then, a great drop on the network performance at $500 \mathrm{~mW}$ (Fig. 4.d). 


\section{Macrothink}

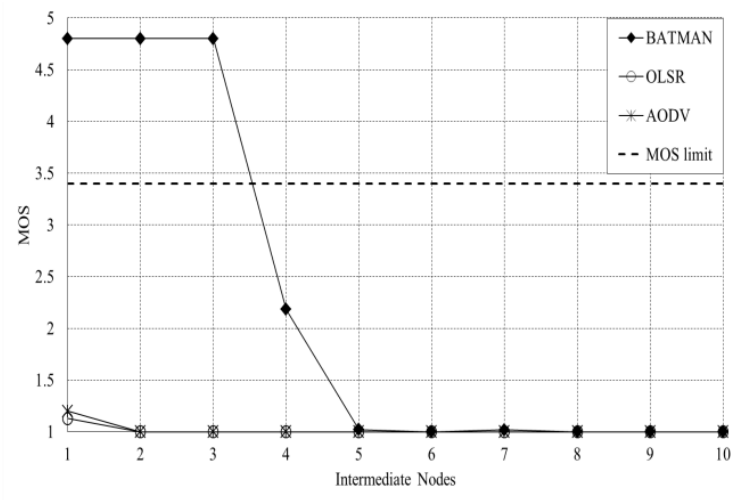

(a) $20 \mathrm{~mW}$

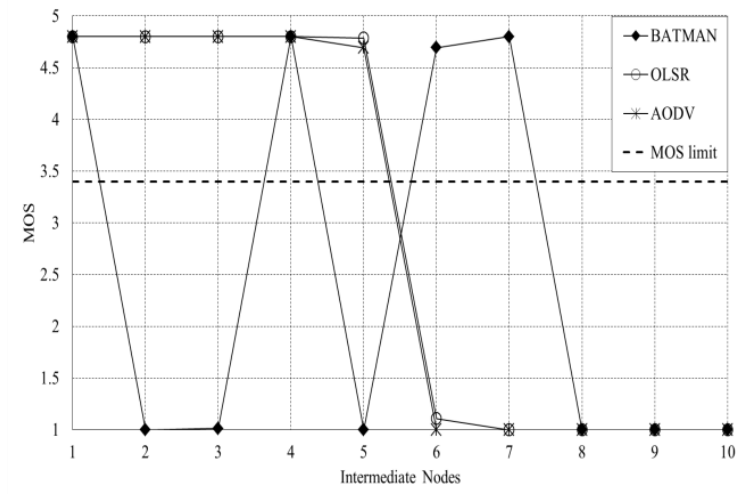

(c) $300 \mathrm{~mW}$

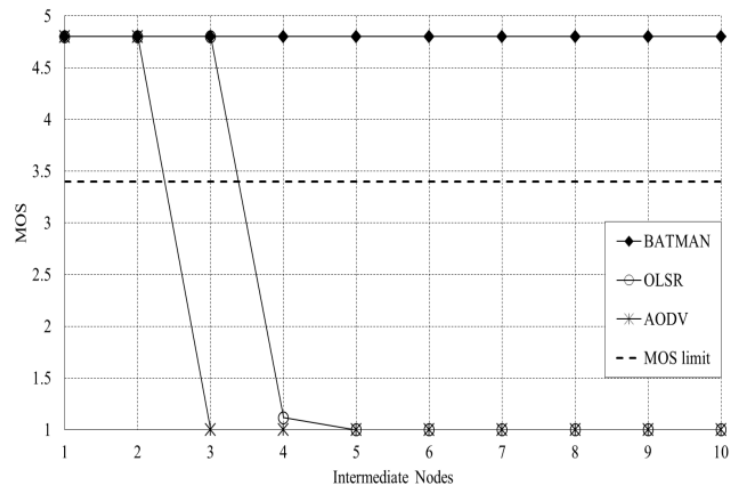

(b) $100 \mathrm{~mW}$

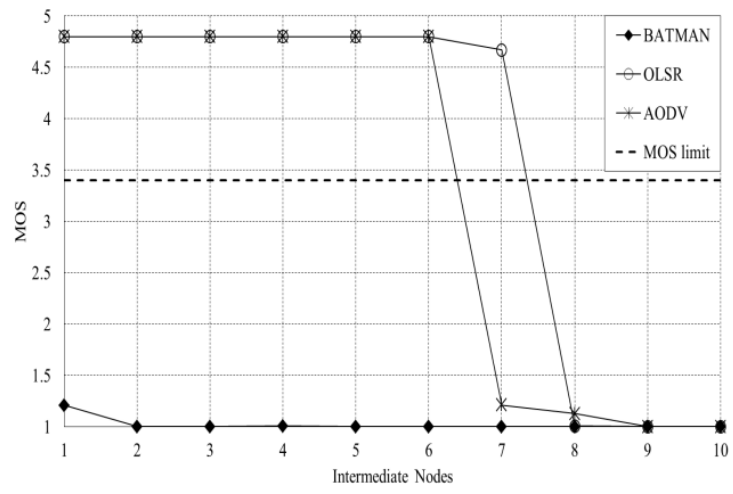

(d) $500 \mathrm{~mW}$

Figure 4. MOS attained in Nakagami-m scenarios for two simultaneous video-streams and variable number of hops between transmitters and receivers, employing different transmission powers. Results for $20 \mathrm{~mW}$ (a), $100 \mathrm{~mW}$ (b), $200 \mathrm{~mW}$ (c), and $500 \mathrm{~mW}$ (d).

We ascribe this behavior to the uncontrolled number of OGMs flooding the network. By increasing the transmission power, the chain topology becomes a linear mesh network where the nodes have more direct neighbors. Thus, the OGMs reach more nodes that retransmit them and so on, which finally causes the network collapse. A deeper discussion about this BATMAN issue is provided in the next sub-section.

\section{Improvement from the Network Layer}

One possible action to improve the quality of the multimedia service running over the network is adapting the routing protocol to the network topology and service characteristics. We ascribe the decay of BATMAN performance obtained in Free Space scenarios (see Fig. 2.a and 2.b) and when the transmission-power is incremented (Fig. 4) to the great amount of control packets (OGMs) that this routing algorithm employs. As the Free Space model does not introduce significant level of BER (Bit Error Rate), the PLR suffered in the transmissions is quite low; so that, the OGMs fully flood the network, collapsing the nodes buffers and obstructing the access to the medium. Thus, in order to improve the video-streams quality, we propose to change the default time interval between OGMs (1 s) by increasing or decreasing it, depending on the network topology and the services running over it. In the chain scenario under evaluation, the nodes are fixed to a static position; hence, it is not necessary the quick refresh of the routing information and the time-interval between OGMs can be enlarged. 


\section{$\triangle$ Macrothink}

Fig. 5 shows the PDR obtained for two simultaneous video-streams with a variable number of intermediate nodes in a low-loss environment, i.e., characterized by the Free Space propagation model. It is clear the great difference between the PDR obtained for the different OGM intervals; in that case, the most proper OGM interval for the chain topology under study is $2 \mathrm{~s}$, meanwhile an excessive time between OGMs, e. g. $5 \mathrm{~s}$, provokes the vast drop on the PDR. Therefore, it is demonstrated how the performance of the multimedia transmission can be noticeably improved by tuning the routing protocols parameters accordingly to the network characteristics.

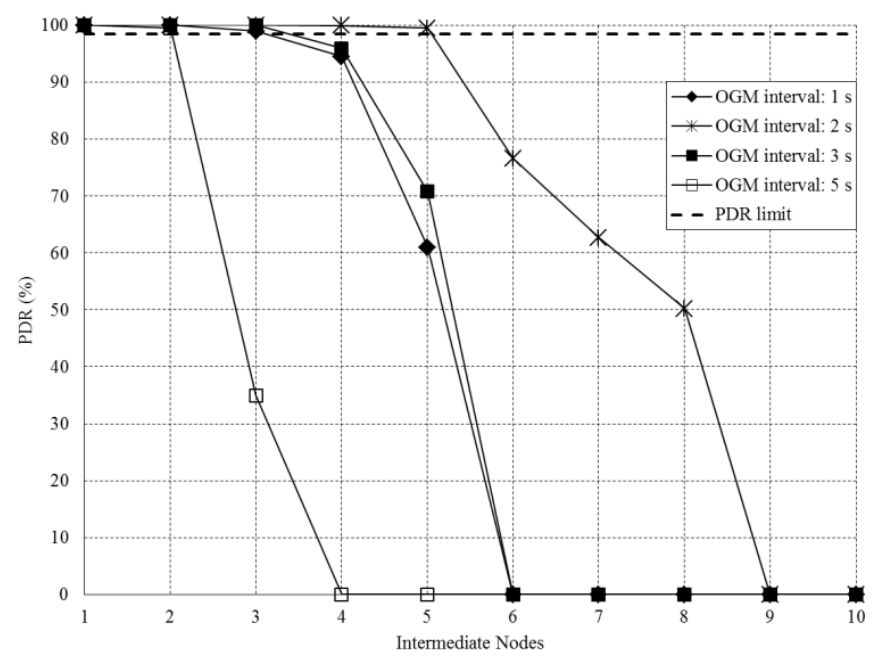

Figure 5. Packet Delivery Ratio attained for two simultaneous video-streams in a Free Space scenario, employing 4 different BATMAN OGM time intervals.

\section{Improvement from the Application layer}

The multimedia coder is another key piece to optimize the performance of the multimedia transmission. Several parameters, such as the coding scheme, the coding rate, or the packetization interval, should be taken into consideration and adapted to the network characteristics. We have evaluated the performance of the system by extending the default coding packetization, i.e., employing a video-packet aggregation of 512 Bytes. Thus, Fig. 6 depicts the MOS obtained in the six scenarios under study. Observe the noticeable improvement in the quality of the transmissions, which in some scenarios makes the MOS to be over the established limit of 3.4 for a greater number of intermediate hops in comparison with the no-aggregation original configuration (Fig. 2).

As an example, observe the overall improvement in Free Space OLSR scenarios (Figs 2.b vs. Fig. 6.b) or the case of two simultaneous video-streams in Nakagami-m scenarios managed by BATMAN (Fig. 2.d and Fig 2.d): whereas the MOS is over the 3.4 limit up to 3 hops without packet-aggregation, using packet-aggregation the communication pass through 5 intermediate hops without trespassing the established PDR threshold. However, the greatest improvement is attained for AODV in Free Space environment. Comparing Fig. 2.e and Fig. 6.e, it is clear the overall rise in the MOS obtained for every simulated scenario.

In the light of the obtained results, we observe that each routing protocol presents different 
response when applying the different actions proposed. Meanwhile OLSR shows the greatest improvement with a properly tuning of the transmission power (Physical Layer), AODV present its best performance by employing video-packet aggregation (Application Layer). We have also shown an enhancement which permits the increasing in the quality of the communication by adjusting the interval between OGMs in BATMAN protocol (Network Layer).

\section{Conclusion}

In this work we have presented the obtained results from the performance evaluation of the novel routing protocol BATMAN and the widely employed OLSR and AODV protocols supporting multimedia traffic in a wireless ad hoc network. Concretely, we have employed the wireless technology 802.11g and we have evaluated several QoS and QoE metrics in a chain-topology network with video-streaming traffic. First, we showed the strength of BATMAN in heavy loss environments, like those characterized by the Nakagami-m model. On the other hand, OLSR and AODV suffer a big drop in their performance in these environments given the greater size of its control packets, which are more affected by the fading channels. However, in Free Space scenarios, i.e., under low-loss conditions, BATMAN presents a degraded performance due to the excess of OGM messages in the network, which causes the system saturation and the rise of the multimedia-packets losses.

Then, we have proposed a number of actions, taken from different OSI layers that permit a noticeable improvement of the multimedia quality. Regarding the Physical Layer, we have adjusted the transmission power of the wireless cards to increase the SNiR in hostile scenarios, obtaining different behavior for the three routing protocols; meanwhile AODV and OLSR, especially the latter, improve their performance with greater levels of transmission power, BATMAN suffers a saturation of OGM packets that provokes the drop on the multimedia quality with augmented transmission ranges. From the Network Layer perspective, we have focused on BATMAN, improving its performance by tuning the interval between routing packets, obtaining promising results in order to adapt this protocol to the network and service conditions. Finally, from the Application Layer we have proposed a video-packet aggregation to reduce the number of video packets on the network, obtaining good results for the three protocols, especially for AODV. To sum up, we have shown the strengths and weakness of three different ad-hoc routing protocols which allow the establishment of multimedia communications with quite decent level of quality depending on the environmental conditions and the network characteristics. We consider that, with the aim of improving the performance of multimedia services over ad-hoc systems, the use of cross-layer techniques could be the trend to follow. As shown in this paper, a proper action in the correct OSI layer depending on the environment under study and the impairments affecting the transmission could suppose a significant improvement in the QoE of the multimedia communication. As future work, we plan to extend these inquiries with experimental studies to further complete the results presented in this paper. 


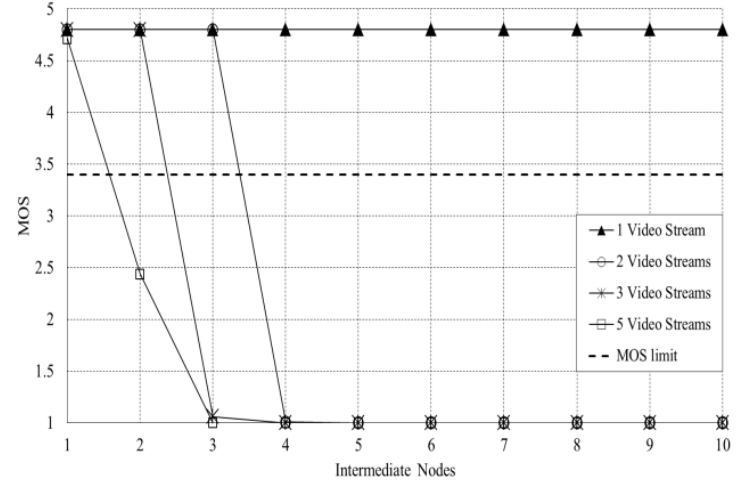

(a) BATMAN. Free Space

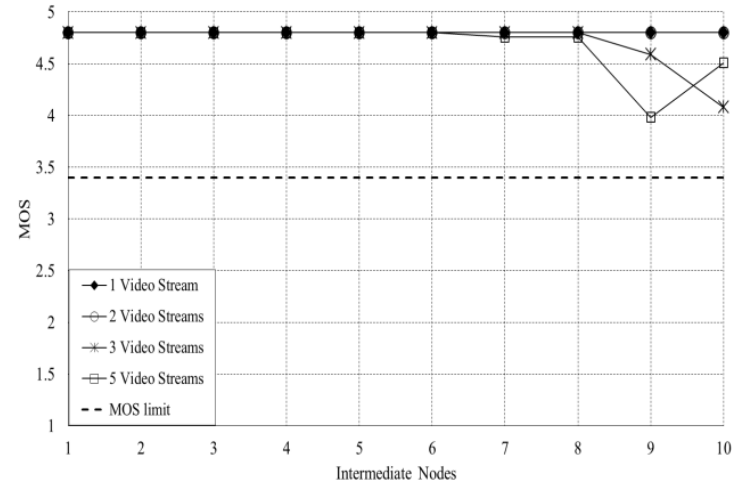

(c) OLSR. Free Space

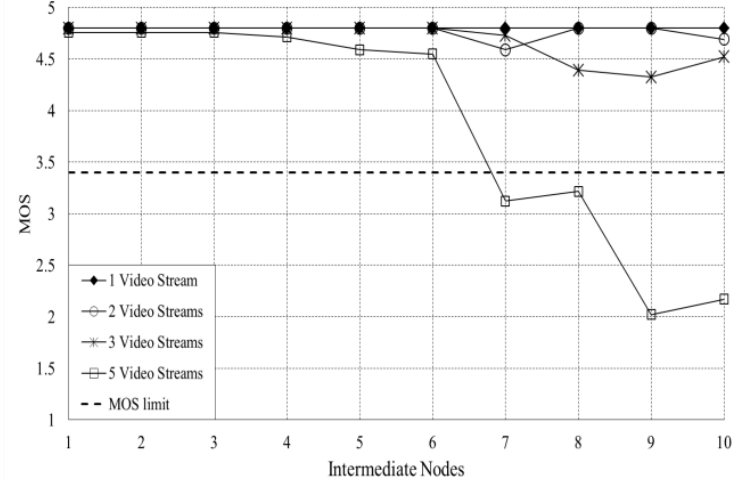

(e) AODV. Free Space

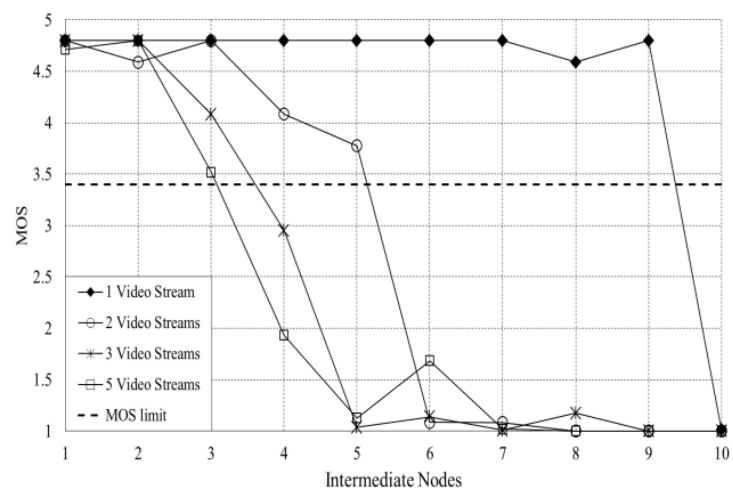

(b) BATMAN. Nakagami-m

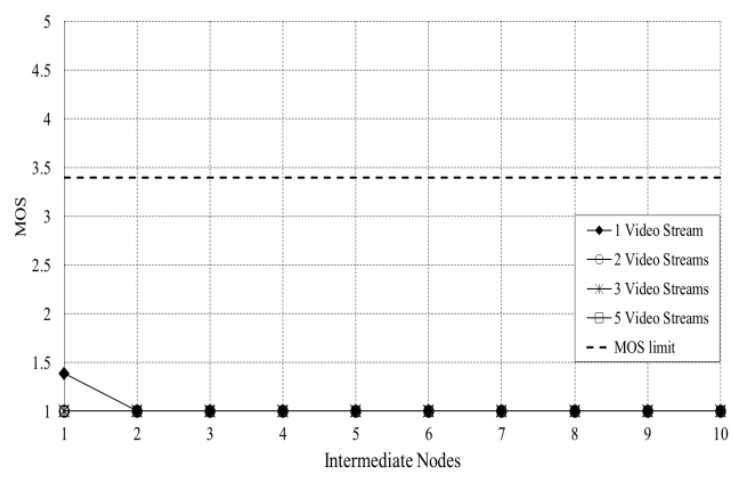

(d) OLSR. Nakagami-m

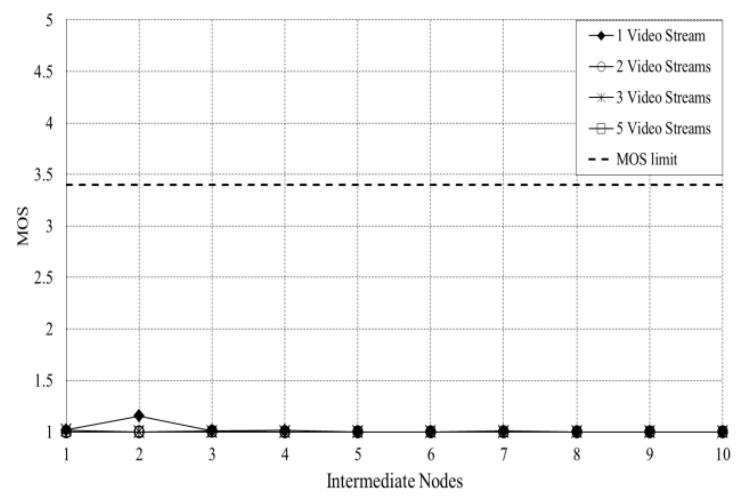

(f) AODV. Nakagami-m

Figure 6. MOS attained for a variable number of video-streams and hops between transmitters and receivers, employing a video-packet aggregation of 512 Bytes. Results for BATMAN in Free Space (a) and Nakagami-m scenarios (b), OLSR in Free Space (c) and Nakagami-m scenarios (d), and AODV in Free Space (e) and Nakagami-m scenarios (f).

\section{Acknowledgement}

This work was supported by the MINECO/FEDER project grant TEC2010-21405-C02-02/TCM (CALM) and "Programa de Ayudas a Grupos de Excelencia de la RM, de la Fundación Séneca, Agencia de Ciencia y Tecnología de la RM". 


\section{References}

[1] S. Shin and H. Schulzrinne, "Measurement and analysis of the VoIP capacity in IEEE 802.11 WLAN," IEEE Trans. Mob. Comput., vol. 8, no. 9, pp. 1265-1279, Sep. 2009, DOI: http://dx.doi.org/10.1109/TMC.2009.49.

[2] R. Sanchez-Iborra, M.-D. Cano, and J. Garcia-Haro, "On the impact of Rice and Rayleigh channel characterization on VoIP services: a QoE approach," Recent Adv. Commun. Netw. Technol., vol. 2, no. 2, 2013, DOI: 10.2174/22117407112016660010.

[3] D. Ferreira, R. A. Costa, and J. Barros, "Real-Time network coding for live streaming in hyper-dense WiFi spaces," IEEE J. Sel. Areas Commun., vol. 32, no. 4, pp. 773-781, Apr. 2014, DOI: http://dx.doi.org/10.1109/JSAC.2014.140409.

[4] A. Neumann, C. Aichele, M. Lindner, and S. Wunderlich, "Better Approach To Mobile Ad-hoc Networking (B.A.T.M.A.N.)," IETF Draft, 2008.

[5] T. Clausen and P. Jacquet, "Optimized Link State Routing (OLSR) Protocol," RFC 3626, 2003.

[6] C. Perkins, E. Belding-Royer, and S. Das, "Ad hoc On-Demand Distance Vector (AODV) Routing," RFC 3561, 2003.

[7] M. Reineri, C. Casetti, and C.-F. Chiasserini, "Routing protocols for mesh networks with mobility support," in 6th International Symposium on Wireless Communication Systems, 2009, pp. 71-75, DOI: http://dx.doi.org/10.1109/ISWCS.2009.5285344.

[8] M. Reineri, R. Rubino, C. Casetti, and C.-F. Chiasserini, "Experimental performance assessment of WMN routing protocols with mobile nodes," in 2011 7th International Wireless Communications and Mobile Computing Conference, 2011, pp. 1010-1015, DOI: http://dx.doi.org/10.1109/IWCMC.2011.5982679.

[9] G. C. Sai Anand, R. R. Vaidya, and T. Velmurugan, "Performance analysis of VoIP traffic using various protocols and throughput enhancement in WLANs," in International Conference on Computer, Communication and Electrical Technology, 2011, pp. 176-180, DOI: http://dx.doi.org/10.1109/ICCCET.2011.5762463.

[10] R. K. Gujral and M. Singh, "Performance analysis of ad hoc routing protocols for voice communication support over hybrid MANETs," Int. J. Comput. Appl., vol. 22, no. 3, pp. 42-48, 2011, DOI: http://dx.doi.org/10.5120/2560-3515.

[11]E. Kulla, M. Ikeda, T. Oda, L. Barolli, F. Xhafa, and M. Takizawa, "Multimedia Transmissions over a MANET Testbed: Problems and Issues," in Sixth International Conference on Complex, Intelligent, and Software Intensive Systems, 2012, pp. 141-147, DOI: http://dx.doi.org/10.1109/CISIS.2012.82. 
[12] G. Adam, C. Bouras, A. Gkamas, V. Kapoulas, G. Kioumourtzis, and N. Tavoularis, "Performance evaluation of routing protocols for multimedia transmission over mobile ad hoc networks," in 4th Joint IFIP Wireless and Mobile Networking Conference (WMNC 2011), 2011, pp. 1-6, DOI: 10.1109/WMNC.2011.6097250.

[13]F. Community, "Better Approach To Mobile Ad hoc Networking (B.A.T.M.A.N.)." [Online]. Available: http://www.open-mesh.org/.

[14] D. Johnson, N. Ntlatlapa, and C. Aichele, "A simple pragmatic approach to mesh routing using BATMAN," in 2nd IFIP International Symposium on Wireless Communications and Information Technology in Developing Countries, 2008, DOI: http://hdl.handle.net/10204/3035.

[15] “Omnet++.” [Online]. Available: <www.omnetpp.org>.

[16] "Video Trace Library." [Online]. Available: <http://trace.eas.asu.edu/>.

[17]K. Yamagishi and T. Hayashi, "Parametric packet-layer model for monitoring video quality of IPTV services," in IEEE International Conference on Communications, 2008, pp. 110-114, DOI: 10.1109/ICC.2008.29.

\section{Copyright Disclaimer}

Copyright reserved by the author(s).

This article is an open-access article distributed under the terms and conditions of the Creative Commons Attribution license (http://creativecommons.org/licenses/by/3.0/). 